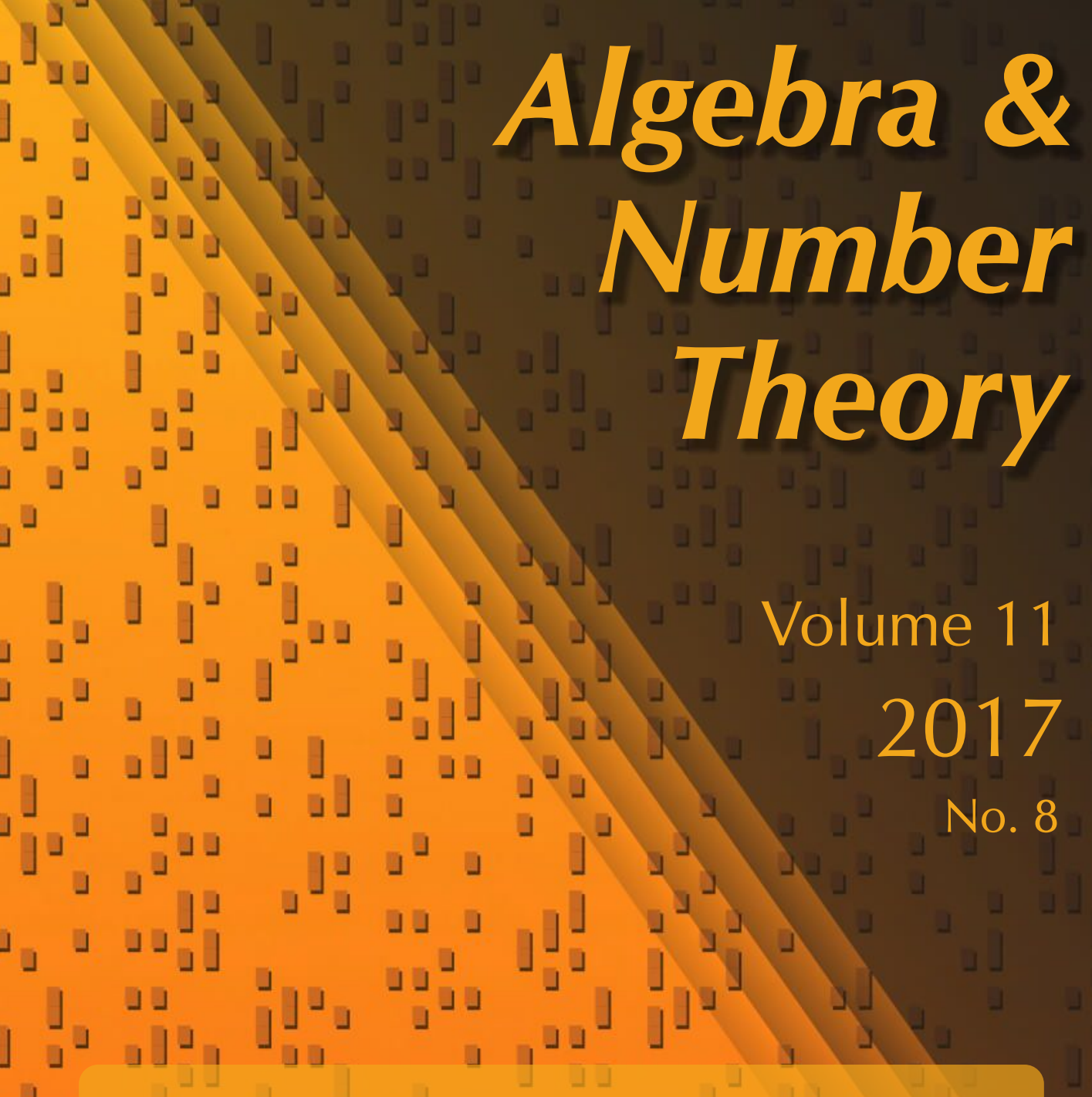

A Mordell-Weil theorem for cubic hypersurfaces of high dimension

Stefanos Papanikolopoulos and Samir Siksek

\lrcorner

. 


\title{
A Mordell-Weil theorem for cubic hypersurfaces of high dimension
}

\author{
Stefanos Papanikolopoulos and Samir Siksek
}

\begin{abstract}
Let $X / \mathbb{Q}$ be a smooth cubic hypersurface of dimension $n \geq 1$. It is well-known that new rational points may be obtained from old ones by secant and tangent constructions. In view of the Mordell-Weil theorem for $n=1$, Manin (1968) asked if there exists a finite set $S$ from which all other rational points can be thus obtained. We give an affirmative answer for $n \geq 48$, showing in fact that we can take the generating set $S$ to consist of just one point. Our proof makes use of a weak approximation theorem due to Skinner, a theorem of Browning, Dietmann and Heath-Brown on the existence of rational points on the intersection of a quadric and cubic in large dimension, and some elementary ideas from differential geometry, algebraic geometry and numerical analysis.
\end{abstract}

\section{Introduction}

Let $X \subset \mathbb{P}^{n+1}$ be a smooth cubic hypersurface over $\mathbb{Q}$ of dimension $n$. Let $\ell \subset \mathbb{P}^{n+1}$ be a line defined over $\mathbb{Q}$. If $\ell$ is not contained in $X$ then $\ell \cdot X=P+Q+R$ where $P, Q, R \in X$. If any two of $P, Q, R$ are rational then so is the third. If $S \subseteq X(\mathbb{Q})$, we write $\operatorname{Span}(S)$ for the subset of $X(\mathbb{Q})$ generated from $S$ by successive secant and tangent constructions. More formally, we define a sequence

$$
S=S_{0} \subseteq S_{1} \subseteq S_{2} \subseteq \cdots \subseteq X(\mathbb{Q})
$$

by letting $S_{n+1}$ be the set of points $R \in X(\mathbb{Q})$ such that either $R \in S_{n}$, or for some $\mathbb{Q}$-line $\ell \not \subset X$ we have $\ell \cdot X=P+Q+R$ where $P, Q \in S_{n}$. Then $\operatorname{Span}(S):=\bigcup S_{n}$. Manin [1974, page 3] (see also [Kanevsky and Manin 2001] and [Manin 1997]) asks if there is some finite subset $S \subset X(\mathbb{Q})$ such that $\operatorname{Span}(S)=X(\mathbb{Q})$.

Theorem 1. Let $X$ be a smooth cubic hypersurface of dimension $n \geq 48$ defined over $\mathbb{Q}$. Then there exists a point $A \in X(\mathbb{Q})$ such that $\operatorname{Span}(A)=X(\mathbb{Q})$.

Siksek is supported by the EPSRC LMF: L-Functions and Modular Forms Programme Grant $\mathrm{EP} / \mathrm{K} 034383 / 1$.

MSC2010: primary 14G05; secondary 11G35.

Keywords: cubic hypersurfaces, rational points, Mordell-Weil problem. 
We are grateful to Tim Browning, Simon Rydin Myerson, Michael Stoll and Damiano Testa for valuable discussions, and to the referees for useful remarks. We thank Yuri Manin for drawing our attention to the distinction between weak and strong Mordell-Weil generation (discussed below).

Some remarks. It appears that the Mordell-Weil problem for cubic surfaces was first posed by Segre [1943]. In the sixties Manin posed the same question for cubic hypersurfaces of arbitrary dimension. Prior to Theorem 1, so far as we know, the only positive result is for cubic surfaces endowed with a skew pair of rational lines [Siksek 2012, Theorem 1]. More recently, Manin [2012] made a distinction between "weak Mordell-Weil generation" where both secant and tangent operations are allowed, and "strong Mordell-Weil generation" where only secant operations are allowed. On the basis of computational experiments carried out by Vioreanu [2009], Manin expects that the weak version of the Mordell-Weil property holds for dimension 2, but that the strong version probably fails. In the language of [Manin 2012], our Theorem 1 establishes the weak Mordell-Weil property for cubic hypersurfaces of dimension $\geq 48$; tangent operations are crucial to our proofs, and we are unable to adapt them to prove the strong Mordell-Weil property.

Notation. Throughout $X \subset \mathbb{P}^{n+1}$ is a smooth cubic hypersurface of dimension $n$ defined over $\mathbb{Q}$ (for now $n \geq 2$ ). Thus there is some non-zero homogeneous cubic polynomial $F \in \mathbb{Q}\left[x_{0}, \ldots, x_{n+1}\right]$ such that $X$ is given by the equation

$$
X: \quad F\left(x_{0}, \ldots, x_{n+1}\right)=0 .
$$

For $P \in X$ we let $T_{P} X$ denote the tangent plane to $X$ at $P$ :

$$
T_{P} X: \quad \nabla F(P) \cdot\left(x_{0}, \ldots, x_{n+1}\right)=0 .
$$

The Gauss map on $X$ sends $P$ to $T_{P} X \in \mathbb{P}^{n+1 *}$. We let $X_{P}:=X \cap T_{P} X$. Thus

$$
X_{P}:\left\{\begin{array}{l}
F\left(x_{0}, \ldots, x_{n}\right)=0, \\
\nabla F(P) \cdot\left(x_{0}, \ldots, x_{n}\right)=0 .
\end{array}\right.
$$

In Section 4 we introduce the second fundamental form $\Pi_{P} X$, and the Hessian $H_{F}(P)$. We write $\mathbb{G}(n+1,1)$ for the Grassmannian parametrizing lines in $\mathbb{P}^{n+1}$. Throughout the terms "open" and "closed" will pertain to the real topology, unless prefixed by "Zariski".

A sketch of the proof of Theorem 1. We show in Section 5 that if $B \in X(\mathbb{Q})$ is not an Eckardt point then $X_{B}(\mathbb{Q}) \subseteq \operatorname{Span}(B)$ (the definition of Eckardt points is given in Section 4). Fix $B \in X(\mathbb{Q})$ that is non-Eckardt. Given $D \in X(\mathbb{Q})$, we ask if there is $C \in X_{B}(\mathbb{Q})$ such that $D \in X_{C}(\mathbb{Q})$ ? If so, then provided $C$ is non-Eckardt, we 
have $D \in \operatorname{Span}(C) \subseteq \operatorname{Span}(B)$. The answer to this question is positive provided the variety $Y_{B, D} \subset \mathbb{P}^{n+1}$ given by

$$
Y_{B, D}:\left\{\begin{array}{l}
F\left(x_{0}, \ldots, x_{n+1}\right)=0 \\
\nabla F\left(x_{0}, \ldots, x_{n+1}\right) \cdot D=0 \\
\nabla F(B) \cdot\left(x_{0}, \ldots, x_{n+1}\right)=0 .
\end{array}\right.
$$

has a rational point. A theorem of Browning, Dietmann and Heath-Brown allows us to deduce the existence of a rational point under some conditions, the most important being that $n$ is large, and that $Y_{B, D}$ has a smooth real point. By considering the second fundamental form, and using a theorem on weak approximation for cubic hypersurfaces due to Skinner, we shall show the existence of a point $B \in X(\mathbb{Q})$ and a non-empty open $U \subseteq X(\mathbb{R})$, so that $Y_{B, D}$ has a smooth real point for all $D \in U$. It follows (with a little care) that $U \cap X(\mathbb{Q}) \subseteq \operatorname{Span}(B)$. Once the existence of such a set $U$ is established, we use Mordell-Weil operations to enlarge $U$ and quickly complete the proof of Theorem 1 .

\section{Some results from analytic number theory}

\section{Weak approximation.}

Theorem 2 [Skinner 1997]. Suppose $n \geq 15$. Then X satisfies weak approximation.

This means that $X(\mathbb{Q})$ is dense in $X\left(A_{\mathbb{Q}}\right)$ where $A_{\mathbb{Q}}$ denotes the adeles. It follows that $X(\mathbb{Q})$ is dense in $X(\mathbb{R})$; a fact we use repeatedly in the proof of Theorem 1 .

Corollary 2.1. Suppose $n \geq 15$. Let $U, V \subseteq X(\mathbb{R})$ be disjoint open sets. Let $A^{\prime} \in U$, $B^{\prime} \in V$, and let $\ell^{\prime} \not \subset X$ be an $\mathbb{R}$-line such that $\ell^{\prime} \cdot X=2 A^{\prime}+B^{\prime}$. Then there are $A \in U \cap X(\mathbb{Q}), B \in V \cap X(\mathbb{Q})$ and $a \mathbb{Q}$-line $\ell \not \subset X$ such that $\ell \cdot X=2 A+B$.

Proof. The projectivized tangent bundle $\mathcal{T}_{X}$ of $X$ parametrizes pairs $(P, \ell)$ with $P \in X$ and $\ell$ a line tangent to $X$ at $P$. We make use of the fact that $\mathcal{T}_{X}$ is locally trivial; thus there is a Zariski open $\mathcal{U}$ containing $A^{\prime}$, and a local isomorphism $\varphi: \mathcal{U} \times \mathbb{P}^{n-1} \rightarrow \mathcal{T}_{X}$ such that $\varphi(P, \alpha)=\left(P, \ell_{P, \alpha}\right)$ where $\ell_{P, \alpha}$ is a line tangent to $X$ at $P$. Moreover, as $A^{\prime}$ is real we take $\varphi$ to be defined over $\mathbb{R}$. Let $W=\mathcal{U}(\mathbb{R}) \cap U$ which is necessarily an open neighbourhood of $A^{\prime}$. Let $\alpha \in \mathbb{P}^{n-1}(\mathbb{R})$ so that $\ell^{\prime}=\ell_{A^{\prime}, \alpha}$. By Theorem 2 we can find $\left\{A_{i}\right\} \subset W \cap X(\mathbb{Q})$ converging to $A^{\prime}$. Write $\ell_{i}=\ell_{A_{i}, \alpha}$. Then $\left\{\ell_{i}\right\}$ converges to $\ell^{\prime}$ in $\mathbb{G}(n+1,1)(\mathbb{R})$. In particular, for sufficiently large $i$, the line $\ell_{i}$ meets $V$. Let $A=A_{i} \in U \cap X(\mathbb{Q})$ for any such large $i$. Choosing a line $\ell / \mathbb{Q}$ tangent to $X$ at $A$ that sufficiently approximates $\ell_{i}$ completes the proof.

Intersections of a cubic with a quadric. Let $Q, C \in \mathbb{Q}\left[x_{1}, \ldots, x_{k}\right]$ be a pair of forms of degrees 2 and 3 respectively, such that

$$
Y: \quad C\left(x_{1}, \ldots, x_{k}\right)=0, \quad Q\left(x_{1}, \ldots, x_{k}\right)=0
$$


is a complete intersection $Y \subset \mathbb{P}^{k-1}$. Using the circle method, Browning, Dietmann and Heath-Brown establish various sufficient conditions for $Y$ to have a rational point. We recount one of their theorems [Browning et al. 2015, Theorem 4] which will be essential to our proof of Theorem 1. Define $\operatorname{ord}_{Q}(C)$ as the least nonnegative integer $m$ such that $C=L Q+C^{\prime}$, with linear form $L \in \mathbb{Q}\left[x_{1}, \ldots, x_{k}\right]$, and such that there is a matrix $\boldsymbol{T} \in \mathrm{GL}_{k}(\mathbb{Q})$ with $C^{\prime}\left(\boldsymbol{T}\left(x_{1}, \ldots, x_{k}\right)\right) \in \mathbb{Q}\left[x_{1}, \ldots, x_{m}\right]$.

Theorem 3 (Browning, Dietmann and Heath-Brown). With notation as above, suppose $k \geq 49$ and $\operatorname{ord}_{Q}(C) \geq 17$. If $Y$ has a smooth real point then $Y(\mathbb{Q}) \neq \varnothing$.

Corollary 2.2. Let $f, q, l \in \mathbb{Q}\left[x_{0}, \ldots, x_{n+1}\right]$, be forms of degree $3,2,1$. Write

$$
Z: \quad f\left(x_{0}, \ldots, x_{n+1}\right)=q\left(x_{0}, \ldots, x_{n+1}\right)=l\left(x_{0}, \ldots, x_{n+1}\right)=0
$$

for their common locus of zeros in $\mathbb{P}^{n+1}$. Suppose that

(i) the cubic hypersurface in $\mathbb{P}^{n+1}$ defined by $f$ is smooth;

(ii) $Z$ has a smooth real point;

(iii) $n \geq 48$.

Then $Z$ has a rational point.

Proof. By a non-singular change of variable, we may suppose that $l=x_{0}$. Let

$$
f^{\prime}\left(x_{1}, \ldots, x_{n+1}\right)=f\left(0, x_{1}, \ldots, x_{n+1}\right), \quad q^{\prime}\left(x_{1}, \ldots, x_{n+1}\right)=q\left(0, x_{1}, \ldots, x_{n+1}\right) .
$$

We may therefore consider $Z$ as being given in $\mathbb{P}^{n}$ as the common locus of $f^{\prime}=$ $q^{\prime}=0$. Suppose $\operatorname{ord}_{q^{\prime}}\left(f^{\prime}\right) \leq 16$. Then a further non-singular change of variables allows us to write

$$
f=x_{0} q_{0}+l^{\prime} q^{\prime}+h\left(x_{1}, \ldots, x_{16}\right) .
$$

where $q_{0}$ is a quadratic form, $l^{\prime}$ is a linear form, and $h$ is a cubic form. Now as $n \geq 48$, there is a common zero in $\mathbb{P}^{n+1}$ to

$$
x_{0}=x_{1}=\cdots=x_{16}=l^{\prime}=q_{0}=q^{\prime}=0 .
$$

This gives a singular point on the cubic hypersurface $f=0$ in $\mathbb{P}^{n+1}$ contradicting (i). We may thus suppose that $\operatorname{ord}_{q^{\prime}}\left(f^{\prime}\right) \geq 17$. A similar argument shows that $f^{\prime}=q^{\prime}=0$ defines a complete intersection in $\mathbb{P}^{n}$. By (ii) this intersection has a smooth real point. Applying Theorem 3 with $k=n+1$ completes the proof.

\section{A numerical stability criterion}

Newton-Raphson. We need a rigorous version of the multivariate Newton-Raphson method. The following result is part of Theorem 5.3.2 of [Stoer and Bulirsch 1980]. 
Here $\|\cdot\|$ denotes the usual Euclidean norm (both for vectors and for matrices). For differentiable $\boldsymbol{f}=\left(f_{1}, \ldots, f_{n}\right): \mathbb{R}^{n} \rightarrow \mathbb{R}^{n}$, denote the Jacobian matrix by $J_{f}$ :

$$
J_{f}:=\left(\frac{\partial f_{i}}{\partial x_{j}}\right)_{i, j=1, \ldots, n} .
$$

Theorem 4. Let $\mathcal{C} \subseteq \mathbb{R}^{n}$ be open, $\mathcal{C}_{0}$ be convex such that $\overline{\mathcal{C}_{0}} \subseteq \mathcal{C}$, and let $\boldsymbol{f}: \mathbb{R}^{n} \rightarrow \mathbb{R}^{n}$ be differentiable for all $\boldsymbol{x} \in \mathcal{C}_{0}$ and continuous for all $\boldsymbol{x} \in \mathcal{C}$.

For $\boldsymbol{x}_{0} \in \mathcal{C}_{0}$ let $r, \alpha, \beta, \gamma, h$ be given positive numbers with the following properties:

$$
\mathcal{B}_{r}\left(\boldsymbol{x}_{0}\right):=\left\{\boldsymbol{x}:\left\|\boldsymbol{x}-\boldsymbol{x}_{0}\right\|<r\right\} \subseteq \mathcal{C}_{0}, \quad h:=\alpha \beta \gamma / 2<1, \quad r:=\alpha /(1-h),
$$

and let $\boldsymbol{f}$ satisfy:

(i) $\left\|J_{f}(\boldsymbol{x})-J_{f}(\boldsymbol{y})\right\| \leq \gamma\|\boldsymbol{x}-\boldsymbol{y}\|$ for all $\boldsymbol{x}, \boldsymbol{y} \in \mathcal{C}_{0}$;

(ii) $J_{f}(\boldsymbol{x})^{-1}$ exists and satisfies $\left\|J_{\boldsymbol{f}}(\boldsymbol{x})^{-1}\right\| \leq \beta$ for all $\boldsymbol{x} \in \mathcal{C}_{0}$;

(iii) $\left\|\boldsymbol{f}\left(\boldsymbol{x}_{0}\right) \cdot J_{f}\left(\boldsymbol{x}_{0}\right)^{-1}\right\| \leq \alpha$.

Then beginning at $\boldsymbol{x}_{0}$ each point

$$
\boldsymbol{x}_{k+1}=\boldsymbol{x}_{k}-\boldsymbol{f}\left(\boldsymbol{x}_{k}\right) \cdot J_{\boldsymbol{f}}\left(\boldsymbol{x}_{k}\right)^{-1}, \quad k=0,1,2, \ldots
$$

is well-defined and belongs to $\mathcal{B}_{r}\left(\boldsymbol{x}_{0}\right)$. Moreover the limit $\lim _{k \rightarrow \infty} \boldsymbol{x}_{k}=\boldsymbol{\xi}$ exists, belongs to $\overline{\mathcal{B}_{r}\left(\boldsymbol{x}_{0}\right)}$ and satisfies $\boldsymbol{f}(\boldsymbol{\xi})=\mathbf{0}$.

Stability. For $f \in \mathbb{R}\left[x_{1}, \ldots, x_{n}\right]$ we shall let $\|f\|_{c}$ denote the maximum of the absolute values of the coefficients of $f$.

Lemma 3.1. Let $g_{1}, \ldots, g_{m} \in \mathbb{R}\left[x_{1}, \ldots, x_{n}\right]$ be polynomials with $m \leq n$. Let $\zeta \in \mathbb{R}^{n}$ be a common zero of $g_{1}, \ldots, g_{m}$, such that $\nabla g_{1}(\zeta), \ldots, \nabla g_{m}(\zeta)$ are linearly independent. Let $\varepsilon>0$ be given. There is $\delta>0$ such that if $f_{1}, \ldots, f_{m} \in \mathbb{R}\left[x_{1}, \ldots, x_{n}\right]$ satisfy $\left\|f_{i}-g_{i}\right\|_{c}<\delta$, then there is $\boldsymbol{\xi} \in \mathbb{R}^{n}$ such that

(a) $\xi$ is a common zero to $f_{1}, \ldots, f_{m}$;

(b) $\nabla f_{1}(\xi), \ldots, \nabla f_{m}(\xi)$ are linearly independent;

(c) $\|\xi-\zeta\|<\varepsilon$.

Proof. Choose $\boldsymbol{v}_{m+1}, \ldots, \boldsymbol{v}_{n} \in \mathbb{R}^{n}$ so that $\nabla g_{1}(\zeta), \ldots, \nabla g_{m}(\zeta), \boldsymbol{v}_{m+1}, \ldots, \boldsymbol{v}_{n}$ is a basis. Let

$$
g_{i}(\boldsymbol{x})=\boldsymbol{v}_{i} \cdot(\boldsymbol{x}-\boldsymbol{\zeta}), \quad i=m+1, \ldots, n .
$$

Then $\zeta$ is a common zero to $g_{1}, \ldots, g_{n}$ and $\nabla g_{1}(\zeta), \ldots, \nabla g_{n}(\zeta)$ are linearly independent. Let $\boldsymbol{g}=\left(g_{1}, \ldots, g_{n}\right)$. Then $J_{g}(\zeta)$ is invertible. We shall fix $f_{i}=g_{i}$ for $i=m+1, \ldots, n$, and let $\boldsymbol{f}=\left(f_{1}, \ldots, f_{n}\right)$. We will apply Theorem 4 with $\boldsymbol{x}_{0}=\boldsymbol{\zeta}$. There is some $\delta_{0}>0$ such that if $\left\|f_{i}-g_{i}\right\|_{c}<\delta_{0}$ then $J_{f}\left(\boldsymbol{x}_{0}\right)$ is invertible. 
Choose $0<r_{0} \leq \varepsilon$ so that condition (ii) of the theorem is satisfied for some $\beta>0$, with $\mathcal{C}_{0}=\overline{\mathcal{B}_{r_{0}}\left(\boldsymbol{x}_{0}\right)}$. Condition (i) holds for some $\gamma>0$ by the multivariate Taylor Theorem. Let $\alpha=\left\|\boldsymbol{f}\left(\boldsymbol{x}_{0}\right) \cdot J_{\boldsymbol{f}}\left(\boldsymbol{x}_{0}\right)^{-1}\right\|$, which depends on $\boldsymbol{f}$. Now $\boldsymbol{g}\left(\boldsymbol{x}_{0}\right)=\mathbf{0}$, so clearly if $\delta \rightarrow 0$, then $\alpha \rightarrow 0$. Therefore for sufficiently small $\delta<\delta_{0}$, we have $h:=\alpha \beta \gamma / 2<1$ and $r:=\alpha /(1-h)<r_{0}$. By the theorem, there is $\xi \in \overline{\mathcal{B}_{r}\left(\boldsymbol{x}_{0}\right)}$ such that $\boldsymbol{f}(\boldsymbol{\xi})=\mathbf{0}$. By construction $\boldsymbol{\xi}$ satisfies (a), (b), (c).

\section{Smooth real points on the varieties $Y_{B, D}$.}

Lemma 3.2. Let $B, D^{\prime}$ be points belonging to $X(\mathbb{R})$ such that the variety $Y_{B, D^{\prime}} \subset$ $X \subset \mathbb{P}^{n+1}$ given by (2) has a smooth real point $C^{\prime}$. Let $V \subseteq X(\mathbb{R})$ be an open neighbourhood of $C^{\prime}$. Then there is an open neighbourhood $U \subseteq X(\mathbb{R})$ of $D^{\prime}$, such that for every $D \in U$, the variety $Y_{B, D}$ has a smooth real point $C \in V$.

Proof. We may suppose that $B, C^{\prime}, D^{\prime}$ are contained in the affine patch $x_{0}=1$. Let $G_{1}, G_{2}, G_{3}$ be the three polynomials defining $Y_{B, D^{\prime}}$ in (2) and let $g_{1}, g_{2}$, $g_{3} \in \mathbb{R}\left[x_{1}, \ldots, x_{n+1}\right]$ be their dehomogenizations by $x_{0}=1$. Write $f_{1}, f_{2}, f_{3}$ for the corresponding polynomials in $\mathbb{R}\left[x_{1}, \ldots, x_{n+1}\right]$ defining $Y_{B, D} \cap\left\{x_{0}=1\right\}$ with $D \in X(\mathbb{R}) \cap\left\{x_{0}=1\right\}$. Of course $f_{1}=g_{1}, f_{3}=g_{3}$, and moreover

$$
\left\|f_{2}-g_{2}\right\|_{c} \leq \mu \cdot\left\|D-D^{\prime}\right\|_{\infty}
$$

where $\mu>0$ is a constant and $\|\cdot\|_{\infty}$ denotes the infinity norm in the affine patch $x_{0}=1$ (which we identify with $\mathbb{R}^{n+1}$ ). Now $C^{\prime} \in \mathbb{R}^{n+1}$ is a common zero for $g_{1}, g_{2}, g_{3}$ with $\nabla g_{1}\left(C^{\prime}\right), \nabla g_{2}\left(C^{\prime}\right), \nabla g_{3}\left(C^{\prime}\right)$ linearly independent (as $C^{\prime}$ is now a smooth point on the affine patch $\left.Y_{B, D^{\prime}} \cap\left\{x_{0}=1\right\}\right)$. Let $\varepsilon>0$ be sufficiently small so that $\mathcal{B}_{\varepsilon}\left(C^{\prime}\right) \cap X(\mathbb{R})$ is contained in $V$. Applying Lemma 3.1, we know that if $\left\|D-D^{\prime}\right\|_{\infty}$ is sufficiently small then there is a non-zero vector $C \in \mathcal{B}_{\varepsilon}\left(C^{\prime}\right)$ that is a common zero for $f_{1}, f_{2}, f_{3}$ with $\nabla f_{1}(C), \nabla f_{2}(C), \nabla f_{3}(C)$ linearly independent. This completes the proof.

Lemma 3.3. Suppose $n \geq 48$. Let $B \in X(\mathbb{Q})$. Suppose $D^{\prime} \in X(\mathbb{R})$ such that $Y_{B, D^{\prime}}$ has a smooth real point $C^{\prime}$. Then there is a non-empty open $U \subseteq X(\mathbb{R})$ such that if $D \in U \cap X(\mathbb{Q})$, then $Y_{B, D}(\mathbb{Q}) \neq \varnothing$.

Proof. Let $U$ be as in Lemma 3.2. Then $Y_{B, D}$ is defined over $\mathbb{Q}$ and has a smooth real point for every $D \in U \cap X(\mathbb{Q})$. Now the lemma follows from Corollary 2.2.

\section{A little geometry}

\section{Lines on $X$.}

Lemma 4.1. Let $\ell$ be a line contained in $X$ and $P \in \ell$. Then $\ell \subset T_{P} X$.

This is well-known; for a proof, see [Siksek 2012, Lemma 2.1]. 
The second fundamental form. Let $P \in X$. Associated to $P$ is a quadratic form (well-defined up to multiplication by a non-zero scalar) known as the second fundamental form which we denote by $\Pi_{P} X$, and which is defined as the differential of the Gauss map (e.g., [Griffiths and Harris 1979; Harris 1992, Chapter 17]. For our purpose the following explicit recipe given in [Griffiths and Harris 1979, pages 369-370] is useful. By carrying out a non-singular change of coordinates we may suppose $P$ is $(1: 0: \ldots: 0)$, and the tangent plane $T_{P} X$ to $X$ at $P$ given by $x_{n+1}=0$. Then $X$ has the equation $F=0$ with

$$
F=x_{0}^{2} x_{n+1}+x_{0} q\left(x_{1}, \ldots, x_{n+1}\right)+c\left(x_{1}, \ldots, x_{n+1}\right)
$$

where $q$ and $c$ are homogeneous of degree 2 and 3 respectively. Write $z_{1}=$ $x_{1} / x_{0}, \ldots, z_{n+1}=x_{n+1} / x_{0}$. We can take $z_{1}, \ldots, z_{n}$ as local coordinates for $X$ at $P$, and then $X$ is given by the local equation

$$
z_{n+1}=q^{\prime}\left(z_{1}, \ldots, z_{n}\right)+\text { (higher order terms). }
$$

Here $q^{\prime}\left(z_{1}, \ldots, z_{n}\right)=-q\left(z_{1}, \ldots, z_{n}, 0\right)$. The second fundamental form $\Pi_{P} X$ is the quadratic form $q^{\prime}\left(d z_{1}, \ldots, d z_{n}\right)$ (up to scaling). We shall only be concerned with the rank and signature of $\Pi_{P} X$, which are precisely the rank and signature of $q\left(x_{1}, \ldots, x_{n}, 0\right)$ and so we will take this as the second fundamental form. We may therefore view it as the restriction of $q$ to $T_{P} X$. The following follows easily from the above description and the implicit function theorem.

Lemma 4.2. Suppose $\Pi_{P} X$ has full rank $n$.

(i) If $\Pi_{P} X$ is definite then there is an open neighbourhood $U \subseteq X(\mathbb{R})$ such that $U \cap X_{P}(\mathbb{R})=\{P\}$.

(ii) If $\Pi_{P} X$ is indefinite then for every open neighbourhood $U \subseteq X(\mathbb{R})$ of $P$ the intersection contains a real manifold of dimension $n-1$.

Lemma 4.3. There is a non-empty subset $U_{1} \subseteq X(\mathbb{R})$, open in the real topology, such that for $P \in U_{1}$ the second fundamental form $\Pi_{P} X$ is indefinite of full rank.

Proof. A theorem of Landsberg [1994, Theorem 6.1] asserts that at a general point on smooth hypersurface of degree $\geq 2$, the second fundamental form has full rank. Thus there is a Zariski open $\mathcal{U} \subset X$ such that $\Pi_{P} X$ has full rank for $P \in \mathcal{U}$.

A straightforward application of Bertini's Theorem shows the existence of a real 3-dimensional linear subvariety $\Lambda \subset \mathbb{P}^{n+1}$ such that $X^{\prime}=\Lambda \cap X$ is a smooth real cubic surface. A classical theorem of Schläfli asserts that the number of real lines on smooth real cubic surface is either $3,7,15$ or 27 . Let $\ell \subset \Lambda \cap X$ be a real line. Recall that a point on a smooth real surface is called hyperbolic if the Gaussian curvature at the point is negative. By [Siksek 2012, Lemma 2.2] all but at most two points of $\ell(\mathbb{R})$ are hyperbolic for $X^{\prime}$. Let $Q \in \ell(\mathbb{R})$ be a hyperbolic point for $X^{\prime}$. 
The determinant of the second fundamental form $\Pi_{Q} X^{\prime}$ is the Gaussian curvature of $X^{\prime}$ at $Q$, which is negative. It follows that the binary quadratic form $\Pi_{Q} X^{\prime}$ is indefinite. Now $\Pi_{Q} X^{\prime}$ is the restriction of $\Pi_{Q} X$ to $T_{Q} X^{\prime}$ and so $\Pi_{Q} X$ is indefinite. Thus there is a neighbourhood $V \subseteq X(\mathbb{R})$ of $Q$, open in the real topology, such that $\Pi_{P} X$ is indefinite for $P \in V$. Now $V$ is necessarily Zariski-dense in $X$. Thus $V \cap \mathcal{U}(\mathbb{R})$ is non-empty (as well as being open in the real topology). The proof is complete upon letting $U_{1}=V \cap \mathcal{U}(\mathbb{R})$.

The Hessian. Given $P \in X$, the Hessian of $F$ evaluated at $P$ is given by the $(n+2) \times(n+2)$ matrix

$$
H_{F}(P)=\left(\frac{\partial^{2} F}{\partial x_{i} \partial x_{j}}(P)\right)_{i, j=0, \ldots, n+1} .
$$

Of course the Hessian is well-defined up to multiplication by a non-zero scalar.

Lemma 4.4. Let $P \in X$ and suppose $\Pi_{P}(X)$ has full rank $n$. Then $H_{F}(P)$ has full rank $n+2$.

Proof. Starting from (3), an easy computation shows that the determinant of the Hessian at $P$ is (up to sign) the determinant of $q\left(x_{1}, \ldots, x_{n}, 0\right)$.

Eckardt points. We call $P \in X$ an Eckardt point if $X_{P}:=X \cap T_{P} X$ is a cone with vertex at $P$. Note that if $n=2$ and $P$ is an Eckardt point then $X_{P}$ consists of three lines meeting at $P$; in this case $\Pi_{P} X$ vanishes identically.

For a proof of the following classical theorem see [Coskun and Starr 2009, Section 2].

Theorem 5. The set of Eckardt points on $X$ is finite.

Components of a real cubic hypersurface. We summarize some well-known facts about components of real cubic hypersurfaces. Everything we need is actually contained in [Viro 1998, Section 4.3]. A smooth real cubic hypersurface has either one or two connected components. If it has two connected components then one of these is two-sided, and homeomorphic to $S^{n}$, and the other is one-sided and homeomorphic to $\mathbb{R} P^{n}$. If a line intersects the two-sided component then it intersects it in two points, and intersects the odd-sided component in one point.

Lemma 4.5. Suppose $X(\mathbb{R})$ has two connected components. Then $\Pi_{P} X$ is definite of full rank for all $P$ belonging to the two-sided component.

Proof. Let $P$ be a point on the two-sided component. Suppose $\Pi_{P} X$ is indefinite or not of full rank. Then there is a real line $\ell \subset T_{P} X$ (along which $\Pi_{P} X$ vanishes) that meets $X$ with multiplicity $\geq 3$ at $P$. As this is impossible for points on the two-sided component, we have a contradiction. 


\section{Mordell-Weil generation: first steps}

Proposition 5.1. Let $P \in X(\mathbb{Q})$ be a non-Eckardt point. Then the set $X_{P}(\mathbb{Q})$ (considered as a subset of $X(\mathbb{Q})$ ) is contained in $\operatorname{Span}(P)$.

The following lemma follows from the definitions.

Lemma 5.2. Let $P \in X(\mathbb{Q})$ and let $Q \in X_{P}(\mathbb{Q})$ be distinct from $P$. Suppose the line $\ell$ joining $P$ to $Q$ is not contained in $X$. Then $Q \in \operatorname{Span}(P)$.

For the proof of Proposition 5.1 it remains to show that $Q \in \operatorname{Span}(P)$ in the case $\ell \subset X$. For $n=2$ this is [Siksek 2012, Lemma 3.2], so we suppose for the remainder of this section that $n \geq 3$.

Lemma 5.3. Any hyperplane section of $X$ is absolutely irreducible.

Proof. Let $L=0$ be a hyperplane such that $X \cap\{L=0\}$ is absolutely reducible. Then we can write $F=L Q+L^{\prime} Q^{\prime}$ where $L, L^{\prime}$ are homogeneous linear, and $Q$, $Q^{\prime}$ are homogeneous quadratic. As $n \geq 3$, the variety $L=L^{\prime}=Q=Q^{\prime}=0$ has a point $R \in \mathbb{P}^{n+1}$. It follows that $R$ is a singular on $X$ giving a contradiction.

Lemma 5.4. Let $P \in X(\mathbb{Q})$ be a non-Eckardt point. Let $Q \in X_{P}(\mathbb{Q})$ with $T_{Q} X \neq$ $T_{P} X$. Then $Q \in \operatorname{Span}(P)$.

Proof. Let $\mathcal{W} \subseteq X_{P}$ be the subvariety consisting of lines through $P$ contained in $X_{P}$. As $P$ is a non-Eckardt point, $\mathcal{W}$ is a proper subvariety. Moreover, by Lemma 5.3, the tangent plane section $X_{P}$ is irreducible, and $\operatorname{so} \operatorname{dim}(\mathcal{W})<\operatorname{dim}\left(X_{P}\right)$. Let $\mathcal{U}=X_{P}-\mathcal{W}$ which is Zariski dense in $X_{P}$.

Let $\mathcal{V}:=X_{P} \backslash\left(X_{P} \cap T_{Q}\right)$. As $T_{Q} \neq T_{P}$, this is a dense open subset of $X_{P}$. Let $\iota: \mathcal{V} \rightarrow \mathcal{V}$ be the involution given as follows. If $R \in \mathcal{V}$ we join $R$ to $Q$ by the line $\ell_{R, Q}$ and we let $\iota(R)$ be the third point of intersection of this line with $X$. We note that $\ell_{R, Q} \not \subset X$, since otherwise it will be contained in $T_{Q} X$ by Lemma 4.1. Now $(\mathcal{V} \cap \mathcal{U}) \cap \iota(\mathcal{V} \cap \mathcal{U})$ is a Zariski dense subset of the rational variety $X_{P}$. This dense subset must contain a rational point $R$. Then $R, \iota(R) \notin \mathcal{W}$ and so $R, \iota(R) \in \operatorname{Span}(P)$ by Lemma 5.2. Finally the line joining $R$ with $\iota(R)$ passes through $Q$ and is not contained in $X$. Thus $Q \in \operatorname{Span}(R)$.

Proof of Proposition 5.1. Let $Q \in X_{P}(\mathbb{Q})$. We would like to show that $Q \in \operatorname{Span}(P)$. Thanks to Lemmas 5.2 and 5.4, we may suppose there is a $\mathbb{Q}$-line $\ell \subset X$ containing $P, Q$, and $T_{Q} X=T_{P} X$. Now the line $\ell$ contains at most finitely many Eckardt points by Theorem 5. Moreover, the Gauss map on a smooth hypersurface has finite fibres [Harris 1992, Lecture 15]. Thus there is a non-Eckardt $R \in \ell(\mathbb{Q})$ with $T_{R} X \neq T_{P} X$. It follows that $R \in \operatorname{Span}(P)$. Moreover, $Q \in \ell \subset T_{R}$ by Lemma 4.1 and so $Q \in \operatorname{Span}(R)$ (again by Lemma 5.4). This completes the proof. 
Lemma 5.5. Suppose $n \geq 48$. Let $B \in X(\mathbb{Q})$ so that $X_{B}$ does not contain points that are Eckardt for $X$. Suppose $D^{\prime} \in X(\mathbb{R})$ such that $Y_{B, D^{\prime}}$ has a smooth real point $C^{\prime}$. Then there is a non-empty open $U \subseteq X(\mathbb{R})$ such that $U \cap X(\mathbb{Q}) \subseteq \operatorname{Span}(B)$.

Proof. Take $U$ to be as in Lemma 3.3. Let $D \in U \cap X(\mathbb{Q})$. By the conclusion of Lemma 3.3 we see that $Y_{B, D}$ has a rational point $C$. From the equations defining $Y_{B, D}$ in (2) we have that $C \in X_{B}(\mathbb{Q})$ and $D \in X_{C}(\mathbb{Q})$. Moreover, neither $B$ nor $C$ (both contained in $X_{B}$ ) are Eckardt points. Applying Proposition 5.1, we have $C \in \operatorname{Span}(B)$ and $D \in \operatorname{Span}(C)$ completing the proof.

\section{A smoothness criterion}

Lemma 6.1. Let $B \in X$, Let $C^{\prime} \in X_{B}$ and $D^{\prime} \in X_{C^{\prime}}$. Suppose

(i) $T_{C^{\prime}} X \neq T_{B} X$;

(ii) $H_{F}\left(C^{\prime}\right)$ has full rank, where $H_{F}$ is the Hessian matrix;

(iii) $D^{\prime}$ does not belong to the line

$$
\left\{\left(\lambda \nabla F(B)+\mu \nabla F\left(C^{\prime}\right)\right) \cdot H_{F}\left(C^{\prime}\right)^{-1} \quad: \quad(\lambda: \mu) \in \mathbb{P}^{1}\right\} .
$$

Then $C^{\prime}$ is a smooth point on the variety $Y_{B, D^{\prime}} \subset \mathbb{P}^{n+1}$ given by (2).

Proof. As $C^{\prime} \in X_{B}$ and $D^{\prime} \in X_{C^{\prime}}$ we see that $C^{\prime} \in Y_{B, D^{\prime}}$. We need to show that $C^{\prime}$ is a smooth point on $Y_{B, D^{\prime}}$. Write

$$
f\left(x_{0}, \ldots, x_{n+1}\right)=\nabla F\left(x_{0}, \ldots, x_{n+1}\right) \cdot D^{\prime}, \quad g=\nabla F(B) \cdot\left(x_{0}, \ldots, x_{n+1}\right) .
$$

To show that $C^{\prime}$ is smooth on $Y_{B, D^{\prime}}$ it is enough to show that $\nabla F\left(C^{\prime}\right), \nabla f\left(C^{\prime}\right)$ and $\nabla g\left(C^{\prime}\right)$ are linearly independent. A straightforward computation shows that

$$
\nabla f\left(C^{\prime}\right)=D^{\prime} \cdot H_{F}\left(C^{\prime}\right), \quad \nabla g\left(C^{\prime}\right)=\nabla F(B) .
$$

Suppose

$$
\varepsilon D^{\prime} \cdot H_{F}\left(C^{\prime}\right)+\lambda \nabla F(B)+\mu \nabla F\left(C^{\prime}\right)=0 .
$$

By assumptions (ii) and (iii) we see that $\varepsilon=0$. However, $\nabla F(B)$ and $\nabla F\left(C^{\prime}\right)$ are linearly independent by assumption (i), and so $\lambda=\mu=0$.

\section{Proof of Theorem 1}

In this section $n \geq 48$.

Lemma 7.1. There is $A \in X(\mathbb{Q})$ and a non-empty open $U \subseteq X(\mathbb{R})$ such that:

(i) $U \cap X(\mathbb{Q}) \subseteq \operatorname{Span}(A)$;

(ii) $\operatorname{Span}(A)$ contains at least one point in every connected component of $X(\mathbb{R})$. 
Proof. Suppose first that $X(\mathbb{R})$ is connected. Let $U_{1} \subseteq X(\mathbb{R})$ be the non-empty open subset whose existence is guaranteed by Lemma 4.3: for every $P \in U_{1}$, the second fundamental form $\Pi_{P} X$ is indefinite of full rank. It follows from Theorem 5 that the set of points $P$ with $X_{P}$ containing an Eckardt point is a proper subset of $X$ that is closed in the Zariski topology. Thus we may replace $U_{1}$ by a non-empty open set $U_{2} \subseteq U_{1}$ such that for every $P \in U_{2}$, the subvariety $X_{P}$ does not contain points that are Eckardt for $X$. Fix $B \in U_{2} \cap X(\mathbb{Q})$ whose existence is guaranteed by Theorem 2. The hypersurface $X$ is smooth of degree 3, and so the Gauss map $X \rightarrow X^{*}$ has finite fibres [Harris 1992, Lecture 15]. We can therefore take an open neighbourhood $U_{3} \subseteq U_{2}$ of $B$ such that for all $C^{\prime} \in U_{3}$ with $C^{\prime} \neq B$, we have $T_{B} X \neq T_{C^{\prime}} X$. By Lemma 4.2, the intersection $U_{3} \cap X_{B}(\mathbb{R})$ contains a real manifold of dimension $n-1$; choose $C^{\prime} \in U_{3} \cap X_{B}(\mathbb{R})$ with $C^{\prime} \neq B$. As the second fundamental form has full rank on $U_{3}$, we see from Lemma 4.4 that $H_{F}\left(C^{\prime}\right)$ is of full rank $n+2$. Now again by Lemma 4.2, the intersection $U_{3} \cap X_{C^{\prime}}(\mathbb{R})$ contains a manifold of real dimension $n-1$, and so we can find $D^{\prime} \in U_{3} \cap X_{C^{\prime}}(\mathbb{R})$ that avoids the line (4). The points $B, C^{\prime}, D^{\prime}$ satisfy the conditions of Lemma 6.1. Thus $C^{\prime}$ is a smooth point on $Y_{B, D^{\prime}}$. By Lemma 5.5, there is a non-empty open $U$ such that $U \cap X(\mathbb{Q}) \subseteq \operatorname{Span}(B)$. We simply take $A=B$, and the proof is complete in the case when $X(\mathbb{R})$ is connected.

Now suppose $X(\mathbb{R})$ has two connected components. Let $U_{2} \subseteq X(\mathbb{R})$ be as above. From Lemma 4.5 we know that $U_{2}$ is contained in the one-sided component. Let $B^{\prime} \in U_{2}$. Let $\ell^{\prime}$ be a real line passing through $B^{\prime}$ and tangent to the two-sided component at a point $A^{\prime}$. By Corollary 2.1 , there is a point $A \in X(\mathbb{Q})$ belonging to the two-sided component and a line $\ell$ defined over $\mathbb{Q}$ such that $\ell \cdot X=2 A+B$ where $B \in U_{2} \cap X(\mathbb{Q})$. Now $B \in \operatorname{Span}(A)$ and $\operatorname{Span}(A)$ contains points belonging to both components of $X(\mathbb{R})$. From the above argument there is a non-empty open $U \subseteq X(\mathbb{R})$ such that $U \cap X(\mathbb{Q}) \subseteq \operatorname{Span}(B) \subseteq \operatorname{Span}(A)$.

Lemma 7.2. Let $A \in X(\mathbb{Q})$ be as in Lemma 7.1. Then there is an open $W \subseteq X(\mathbb{R})$ such that $W \cap X(\mathbb{Q})=\operatorname{Span}(A)$.

Proof. Let $U$ be as in Lemma 7.1. We may suppose $\operatorname{Span}(A) \not \subset U$, otherwise we simply take $W=U$ and there is nothing to prove. Let $P \in \operatorname{Span}(A)$ that does not belong to $U$. By Theorem 2, there is some $P^{\prime} \in U \cap X(\mathbb{Q})$ such that $P^{\prime} \notin T_{P} X$. Let $\ell$ be the line joining $P$ to $P^{\prime}$. The line $\ell$ is not contained in $T_{P} X$ and so, by Lemma 4.1, not contained in $X$. Let $P^{\prime \prime} \in X(\mathbb{Q})$ be the third point of intersection of $\ell$ with $X$. Since $P \in \operatorname{Span}(A)$ and $P^{\prime} \in U \cap X(\mathbb{Q}) \subseteq \operatorname{Span}(A)$, we have $P^{\prime \prime} \in \operatorname{Span}(A)$. Observe that $\ell$ is not contained in the tangent plane of $P^{\prime \prime}$ (for otherwise $\ell$ would be contained in $X$ ). Now there is some non-empty open $U^{\prime} \subset U$ containing $P^{\prime}$ that is disjoint from the tangent plane of $P^{\prime \prime}$. For a point $R \in U^{\prime}$, let $\varphi(R)$ denote the third point of intersection of the (real) line joining $R$ to $P^{\prime \prime}$. Then 
the map $\varphi: U^{\prime} \rightarrow X(\mathbb{R})$ is continuous and injective. By the invariance of domain theorem [Bredon 1993, Corollary IV.19.9], the image $\varphi\left(U^{\prime}\right)$ is open. We shall let $W_{P}=\varphi\left(U^{\prime}\right)$. Clearly $P \in W_{P}$ and $W_{P} \cap X(\mathbb{Q}) \subseteq \operatorname{Span}(A)$. The lemma follows on taking

$$
W=U \cup \bigcup_{P \in \operatorname{Span}(A) \backslash U} W_{P}
$$

Lemma 7.3. Let $W$ be as in Lemma 7.2, and write $\bar{W}$ for its closure. Then $\bar{W}$ is closed under secant operations: if $P, Q \in \bar{W}$ are distinct, and if the line $\ell$ joining them is not contained in $X$, then $R \in \bar{W}$ where $\ell \cdot X=P+Q+R$.

Proof. By Theorem 2 there exist $\left\{P_{k}\right\},\left\{Q_{k}\right\} \subset W \cap X(\mathbb{Q})$, with $P_{k} \neq Q_{k}$, that converge respectively to $P, Q$. Write $F \subset \mathbb{G}(n+1,1)$ for the Fano scheme of lines on $X$. Then the real points of $F$ are closed in $\mathbb{G}(n+1,1)(\mathbb{R})$. As $\ell \notin F(\mathbb{R})$, we see for large enough $k$ that the line $\ell_{k} / \mathbb{Q}$ joining $P_{k}, Q_{k}$ is not contained in $X$. Let $\ell_{k} \cdot X=P_{k}+Q_{k}+R_{k}$. Then $\left\{R_{k}\right\}$ converges to $R$. Moreover, $P_{k}$, $Q_{k} \in W \cap X(\mathbb{Q}) \subseteq \operatorname{Span}(A)$. Hence $R_{k} \in \operatorname{Span}(A) \subset W$ and so $R \in \bar{W}$.

Lemma 7.4. Let $A, W$ be as above. Then $\bar{W}=X(\mathbb{R})$.

Proof. We claim that $\bar{W}$ is open. From that it follows that $\bar{W}$ is a union of connected components of $X(\mathbb{R})$. As $\operatorname{Span}(A) \subset \bar{W}$ contains points from every component, the lemma follows from the claim.

To prove the claim we mimic the argument in the proof of Lemma 7.2. Let $P \in \bar{W}$. Let $P^{\prime} \in W$ such that $P^{\prime} \notin T_{P} X$, and let $\ell$ be the line joining $P$ to $P^{\prime}$. As $\bar{W}$ is closed under secant operations, $P^{\prime \prime} \in \bar{W}$ where $\ell \cdot X=P+P^{\prime}+P^{\prime \prime}$. Now there is some non-empty open $W^{\prime} \subset W$ containing $P^{\prime}$ that is disjoint from the tangent plane of $P^{\prime \prime}$. For a point $R \in W^{\prime}$, let $\varphi(R)$ denote the third point of intersection of the (real) line joining $R$ to $P^{\prime \prime}$. Then the map $\varphi: W^{\prime} \rightarrow X(\mathbb{R})$ is continuous and injective, and thus the image $\varphi\left(W^{\prime}\right)$ is open. Clearly $\varphi\left(W^{\prime}\right)$ contains $P$ and is contained in $\bar{W}$ (as the latter is closed under secant operations).

Proof of Theorem 1. Let $A, W$ be as above. In particular, $\operatorname{Span}(A)=W \cap X(\mathbb{Q})$ and $\bar{W}=X(\mathbb{R})$. We write $\partial W=X(\mathbb{R}) \backslash W$. We note that $\partial W$ is the complement of an open dense set, and therefore nowhere dense.

We want to show that $X(\mathbb{Q})=\operatorname{Span}(A)$. Let $P \in X(\mathbb{Q})$. Then there is a Zariski open $\mathcal{U} \subset X$ and an involution $\iota: \mathcal{U} \rightarrow \mathcal{U}$ that sends $R \in \mathcal{U}$ to the third point of the line joining $R$ to $P$. Choose $R \in \mathcal{U}(\mathbb{R}) \cap X(\mathbb{Q})$ such that $R \notin \partial W \cup \iota(\partial W)$. Then $R, \iota(R) \in \operatorname{Span} A$ and so $P \in \operatorname{Span}(A)$.

\section{References}

[Bredon 1993] G. E. Bredon, Topology and geometry, Graduate Texts in Mathematics 139, Springer, New York, 1993. MR Zbl 
[Browning et al. 2015] T. D. Browning, R. Dietmann, and D. R. Heath-Brown, "Rational points on intersections of cubic and quadric hypersurfaces", J. Inst. Math. Jussieu 14:4 (2015), 703-749. Corrigendum in 14:4 (2015), 751. MR Zbl

[Coskun and Starr 2009] I. Coskun and J. Starr, "Rational curves on smooth cubic hypersurfaces", Int. Math. Res. Not. 2009:24 (2009), 4626-4641. MR Zbl

[Griffiths and Harris 1979] P. Griffiths and J. Harris, "Algebraic geometry and local differential geometry”, Ann. Sci. École Norm. Sup. (4) 12:3 (1979), 355-452. MR Zbl

[Harris 1992] J. Harris, Algebraic geometry: a first course, Graduate Texts in Mathematics 133, Springer, New York, 1992. MR Zbl

[Kanevsky and Manin 2001] D. Kanevsky and Y. Manin, "Composition of points and the MordellWeil problem for cubic surfaces", pp. 199-219 in Rational points on algebraic varieties, edited by E. Peyre and Y. Tschinkel, Progr. Math. 199, Birkhäuser, Basel, 2001. MR Zbl

[Landsberg 1994] J. M. Landsberg, "On second fundamental forms of projective varieties", Invent. Math. 117:2 (1994), 303-315. MR Zbl

[Manin 1974] Y. I. Manin, Cubic forms: algebra, geometry, arithmetic, North-Holland Mathematical Library 4, North-Holland, Amsterdam, 1974. Second edition, 1986. MR Zbl

[Manin 1997] Y. I. Manin, "Mordell-Weil problem for cubic surfaces", pp. 313-318 in Advances in mathematical sciences: CRM's 25 years (Montreal, 1994), CRM Proc. Lecture Notes 11, Amer. Math. Soc., Providence, RI, 1997. MR Zbl

[Manin 2012] Y. I. Manin, "Combinatorial cubic surfaces and reconstruction theorems", pp. 99-118 in Zeta functions in algebra and geometry, edited by A. Campillo et al., Contemp. Math. 566, Amer. Math. Soc., Providence, RI, 2012. MR

[Segre 1943] B. Segre, “A note on arithmetical properties of cubic surfaces”, J. London Math. Soc 18 (1943), 24-31. MR Zbl

[Siksek 2012] S. Siksek, "On the number of Mordell-Weil generators for cubic surfaces", J. Number Theory 132:11 (2012), 2610-2629. MR Zbl

[Skinner 1997] C. M. Skinner, "Forms over number fields and weak approximation", Compositio Math. 106:1 (1997), 11-29. MR Zbl

[Stoer and Bulirsch 1980] J. Stoer and R. Bulirsch, Introduction to numerical analysis, Springer, 1980. MR Zbl

[Vioreanu 2009] B. G. Vioreanu, "Mordell-Weil problem for cubic surfaces, numerical evidence", pp. 223-240 in Arithmetic geometry, edited by H. Darmon et al., Clay Math. Proc. 8, Amer. Math. Soc., Providence, RI, 2009. MR

[Viro 1998] O. Viro, "Mutual position of hypersurfaces in projective space", pp. 161-176 in Geometry of differential equations, edited by A. Khovanskii et al., Amer. Math. Soc. Transl. Ser. 2 186, Amer. Math. Soc., Providence, RI, 1998. MR Zbl

Communicated by Brian Conrad

Received 2016-10-10 Revised 2017-07-13 Accepted 2017-08-11

spapanik21@gmail.com Mathematics Institute, University of Warwick, Coventry, United Kingdom

s.siksek@warwick.ac.uk Mathematics Institute, University of Warwick, Coventry, United Kingdom 


\section{Algebra \& Number Theory}

msp.org/ant

\section{EDITORS}

MANAGING EDITOR

Bjorn Poonen

Massachusetts Institute of Technology

Cambridge, USA

\author{
EDITORIAL BOARD CHAIR \\ David Eisenbud \\ University of California \\ Berkeley, USA
}

BOARD OF EDITORS

Richard E. Borcherds

J-L. Colliot-Thélène

Brian D. Conrad

Samit Dasgupta

Hélène Esnault

Gavril Farkas

Hubert Flenner

Sergey Fomin

Edward Frenkel

Andrew Granville

Joseph Gubeladze

Roger Heath-Brown

Craig Huneke

Kiran S. Kedlaya

János Kollár

Yuri Manin

Philippe Michel

Susan Montgomery

Shigefumi Mori
University of California, Berkeley, USA

CNRS, Université Paris-Sud, France

Stanford University, USA

University of California, Santa Cruz, USA

Freie Universität Berlin, Germany

Humboldt Universität zu Berlin, Germany

Ruhr-Universität, Germany

University of Michigan, USA

University of California, Berkeley, USA

Université de Montréal, Canada

San Francisco State University, USA

Oxford University, UK

University of Virginia, USA

Univ. of California, San Diego, USA

Princeton University, USA

Northwestern University, USA

École Polytechnique Fédérale de Lausanne

University of Southern California, USA

RIMS, Kyoto University, Japan
Martin Olsson

Raman Parimala

Jonathan Pila

Anand Pillay

Michael Rapoport

Victor Reiner

Peter Sarnak

Joseph H. Silverman

Michael Singer

Christopher Skinner

Vasudevan Srinivas

J. Toby Stafford

Pham Huu Tiep

Ravi Vakil

Michel van den Bergh

Marie-France Vignéras

Kei-Ichi Watanabe

Shou-Wu Zhang
University of California, Berkeley, USA

Emory University, USA

University of Oxford, UK

University of Notre Dame, USA

Universität Bonn, Germany

University of Minnesota, USA

Princeton University, USA

Brown University, USA

North Carolina State University, USA

Princeton University, USA

Tata Inst. of Fund. Research, India

University of Michigan, USA

University of Arizona, USA

Stanford University, USA

Hasselt University, Belgium

Université Paris VII, France

Nihon University, Japan

Princeton University, USA

PRODUCTION

production@msp.org

Silvio Levy, Scientific Editor

See inside back cover or msp.org/ant for submission instructions.

The subscription price for 2017 is US $\$ 325 /$ year for the electronic version, and $\$ 520 /$ year ( $\$ 55$, if shipping outside the US) for print and electronic. Subscriptions, requests for back issues and changes of subscriber address should be sent to MSP.

Algebra \& Number Theory (ISSN 1944-7833 electronic, 1937-0652 printed) at Mathematical Sciences Publishers, 798 Evans Hall \#3840, c/o University of California, Berkeley, CA 94720-3840 is published continuously online. Periodical rate postage paid at Berkeley, CA 94704, and additional mailing offices.

ANT peer review and production are managed by EditFLOW ${ }^{\circledR}$ from MSP.

\section{PUBLISHED BY}

mathematical sciences publishers

nonprofit scientific publishing

http://msp.org/

(C) 2017 Mathematical Sciences Publishers 


\section{Algebra \& Number Theory}

\section{Volume $11 \quad$ No. $8 \quad 2017$}

On $\ell$-torsion in class groups of number fields

Jordan Ellenberg, Lillian B. Pierce and Melanie Matchett Wood

Torsion orders of complete intersections

ANDRE Chatzistamatiou and MARC LEVINE

Integral canonical models for automorphic vector bundles of abelian type TOM LOVERING

Quasi-Galois theory in symmetric monoidal categories

BREGJE PAUWELS

$\mathfrak{p}$-rigidity and Iwasawa $\mu$-invariants

ASHay A. Burungale and HaRUzo Hida

A Mordell-Weil theorem for cubic hypersurfaces of high dimension

STEFANOS PAPANIKOLOPOULOS and SAMIR SIKSEK 